DRAFT VERSION MARCH 11, 2021

Typeset using LATE twocolumn style in AASTeX61

\title{
GASP VIII. CAPTURING THE BIRTH OF A TIDAL DWARF GALAXY IN A MERGING SYSTEM AT z 0.05
}

\author{
Benedetta Vulcani, ${ }^{1,2}$ Alessia Moretti, ${ }^{2}$ Bianca M. Poggianti, ${ }^{2}$ Giovanni Fasano, ${ }^{2}$ Jacopo Fritz, ${ }^{3}$ \\ Marco GullieusziK, ${ }^{2}$ Pierre-Alain Duc, ${ }^{4}$ Yara Jaffé, ${ }^{5}$ And Daniela Bettoni ${ }^{2}$
}

\footnotetext{
${ }^{1}$ School of Physics, University of Melbourne, VIC 3010, Australia

${ }^{2}$ INAF - Osservatorio astronomico di Padova, Vicolo Osservatorio 5, IT-35122 Padova, Italy

${ }^{3}$ Instituto de Radioastronomía y Astrofísica, UNAM, Campus Morelia, A.P. 3-72, C.P. 58089, Mexico

${ }^{4}$ Université de Strasbourg, CNRS, Observatoire astronomique de Strasbourg, UMR 7550, F-67000 Strasbourg, France

${ }^{5}$ European Southern Observatory, Alonso de Cordova 3107, Vitacura, Casilla 19001, Santiago de Chile, Chile
}

\begin{abstract}
Within the GAs Stripping Phenomena in galaxies with MUSE (GASP) sample, we identified an ongoing 1:1 merger between two galaxies and the consequent formation of a tidal dwarf galaxy (TDG). The system is observed at $z=0.05043$ and is part of a poor group. Exploiting the exquisite quality of the MUSE/VLT data, we present the spatially resolved kinematics and physical properties of gas and stars of this object and describe its evolutionary history. An old (luminosity weighted age $\sim 2 \times 10^{9} \mathrm{yr}$ ), gas poor, early-type-like galaxy is merging with a younger (luminosity weighted age $\sim 2.5 \times 10^{8} \mathrm{yr}$ ), gas rich, late-type galaxy . The system has a quite strong metallicity gradient, indicative of an early-stage phase. Comparing the spatial extension of the star formation at different epochs, we date the beginning of the merger between $2 \times 10^{7} \mathrm{yr}<\mathrm{t}<5.7 \times 10^{8} \mathrm{yr}$ ago. The gas kinematic pattern reflects that of the late-type object and is distorted in correspondence to the location of the impact . The stellar kinematic instead is more chaotic, as expected for mergers. The gas redistribution in the system induces high levels of star formation between the two components, where we indeed detect the birth of the TDG. This stellar structure has a mass of $\sim 6 \times 10^{9} M_{\odot}$, a radius of $\sim 2 \mathrm{kpc}$ and, even though it has already accreted large quantities of gas and stars, it is still located within the disk of the progenitor, is characterized by a high velocity dispersion, indicating that it is still forming, is dusty and has high levels of star formation ( $\mathrm{SFR} \sim 0.3 M_{\odot} y r^{-1}$ ). This TDG is originated in an early-stage merger, while these structures usually form in more evolved systems.
\end{abstract}

Keywords: galaxies: general — galaxies: evolution — galaxies: formation — galaxies: kinematics and dynamics — galaxies: interactions — galaxies: groups: general 


\section{INTRODUCTION}

According to the hierarchical structure formation paradigm (Blumenthal et al. 1984; Freedman et al. 2001; Efstathiou et al. 2002; Pryke et al. 2002; Spergel et al. 2007), smaller systems merge to form progressively larger ones (White \& Rees 1978; Searle \& Zinn 1978). Mergers therefore are fundamental features in galaxy evolution.

Galaxy merging is expected to have a number of consequences on galaxy evolution. Its exact effect depends on a wide variety of parameters such as collision angles, speeds, relative size/composition and environment. Typically, it drives strong star formation episodes (Barnes \& Hernquist 1991; Mihos \& Hernquist 1996; Larson \& Tinsley 1978; Kennicutt 1998a; Elmegreen 2011), contributes to and regulate the growth of black holes (Kauffmann et al. 2003; Di Matteo et al. 2005; Cox et al. 2006; Schawinski et al. 2009; Combes 2003), and produces morphological transformations (Toomre 1977; Mihos \& Hernquist 1996).

During a major merger (with mass ratios from 1:1 to about 3:1), typical star formation rates (SFRs) are less than $10^{2} \mathrm{M}_{\odot} \mathrm{yr}^{-1}$ (Moster et al. 2011; Hirschmann et al. 2012), but can reach peaks of $10^{3} \mathrm{M}_{\odot} \mathrm{yr}^{-1}$, depending on the gas content of each galaxy and its redshift (e.g., Brinchmann et al. 2004; Ostriker \& Shetty 2011). Recent high-resolution models forecast that extended star formation is important in the early stages of the merger, while nuclear starbursts will occur in advanced stages (Teyssier et al. 2010; Hopkins et al. 2013; Renaud et al. 2015). The fast exhaustion of gas ( $<$ few Gyr) during the merger induces the quenching of the star formation and the consequent redistribution of the angular momentum and the violent relaxation in the stellar component, with the transformation of galaxies into an early-type system.

The higher star formation efficiency, along with tidal fields, gravity torques leading to central inflows and outer outflows, increased turbulence, and high Jeans masses induce the formation of a large variety of stellar structures, such as kinematically decoupled cores, super star cluster (SSCs) and tidal dwarf galaxies (TDGs) (e.g., Bournaud 2010).

Simulations by Elmegreen et al. (1993) show that SSCs form from local gravitational instabilities. They can have a similar origin in tidal tails and in isolated systems. These giant molecular clouds form with very high pressures and high gas densities (Li et al. 2004; Bournaud et al. 2008) and can reach a mass of $\sim 10^{6} M_{\odot}$. The star formation efficiency is expected to be very high, so that these SSCs may likely remain bound after the expulsion of gas by the first generations of supernovae, and evolve into globular clusters (Bournaud et al. 2008; Recchi et al. 2007; Renaud et al. 2015).

TDGs form instead only in interacting galaxies, where some disk material become bound and star-forming as a consequence of a "pile-up" in some particular regions. A dense region in a disk (proto-cloud) can be moved outwards as part of a tidal tail. There it can remain bound instead of fragmenting into an unbound complex of several smaller pieces, because of the increased velocity dispersion (Elmegreen et al. 1993; Duc et al. 2004). These massive $\left(\sim 10^{8} M_{\odot}\right)$ star forming structures are made-up mostly of gas and new stars formed locally. The pre-existing stars, present in the parent galaxies before the collision, generally have a too high random velocities, and escape these newly-formed system. TDGs are therefore kinematically decoupled from their parent galaxy, even though gravitationally bound (Bournaud et al. 2007; Duc 2012).

Many TDGs form around or above the critical mass for dwarf galaxies to survive the star formation feedback produced by their initial starburst (Dekel \& Silk 1986; Boquien et al. 2007). Simulations by Bournaud \& Duc (2006) show that a significant fraction of TDGs can survive several Gyrs, orbiting as satellite galaxies around the merger remnant. They may thus contribute to the population of dwarf satellites (Okazaki \& Taniguchi 2000; Metz \& Kroupa 2007).

According to the merger simulations by Bournaud et al. (2008); Chapon et al. (2013), local gravitational instabilities and the pile-up of large amounts of gas can happen at the same time. A single merger rarely form more than a couple of objects through the pile-up mechanism, while many more through instabilities.

Integral field spectroscopy can provide relevant information to characterize merging systems and the resulting stellar structures, since it allows to spatially resolve the properties of the components and to characterize the extent of star formation, and how/when it is produced during the merger event. The observational characterization of star formation in mergers at different stages is also necessary to test the validity and put constraints on merger simulations.

In the last few years, a number of specific interacting/merging systems in the local universe have been observed with such techniques at optical wavelengths (e.g., Wild et al. 2014; Fernández et al. 2015; Cortijo-Ferrero et al. 2017a,c,b). Cortijo-Ferrero et al. (2017c) have also characterized the star cluster properties in an early-stage merger. All these systems are at $z \leq 0.04$ and have been drawn from the CALIFA (Sánchez et al. 2012) and MaNGA (Bundy et al. 2015) surveys, which are characterized by a spatial sampling of $1^{\prime \prime} /$ pixel and 2." 5/pixel and a resolution of $\sim 80 \mathrm{~km} \mathrm{~s}^{-1}$ and $\sim 70 \mathrm{~km} \mathrm{~s}^{-1}$, respectively.

A number of studies based on integral field spectroscopy have also given us some insight into the properties of dwarf galaxies (Izotov et al. 2006; Lagos et al. 2009, 2012, 2014; James et al. 2009, 2010, 2013a,b).

This paper presents the analysis of a merging system at $z \sim$ 0.05 , observed with the integral-field spectrograph MUSE mounted at the VLT, which is currently the most powerful instrument of this kind, having a spatial sampling of $\sim$ 
$0 . / 2 /$ pixel and a resolution of $\sim 50 \mathrm{~km} \mathrm{~s}^{-1}$. The exquisite data quality allows us to detect the formation of a TDG candidate in correspondence of the impact between the two merging components. This is the highest redshift TDG detected up to date. To our knowledge, only Fensch et al. (2016) have exploited the unprecedented spatial resolution of MUSE observations to characterize the physical conditions where a dwarf galaxy formed in a giant collisional HI ring at a distance of 63.1 Mpc.

The system has been observed in the context of GASP ${ }^{1}$ (GAs Stripping Phenomena in galaxies with MUSE), an ongoing ESO Large programme granted 120 hours of observing time with MUSE. The program is aimed at characterizing where, how and why gas can get removed from galaxies. Poggianti et al. (2017, Paper I) presents a complete description of the survey strategy, data reduction and analysis procedures.

The galaxy we discuss here was selected for presenting a B-band morphological asymmetry. Only the integral field spectroscopy allowed us to unambiguously identify the merging event. Note that this system is so far the only merger detected in the survey, indicating that usually signatures of mergers and stripping are distinct.

Throughout all the papers of the GASP series, we adopt a Chabrier (2003) initial mass function (IMF) in the mass range $0.1-100 \mathrm{M}_{\odot}$. The cosmological constants assumed are $\Omega_{m}=0.3, \Omega_{\Lambda}=0.7$ and $\mathrm{H}_{0}=70 \mathrm{~km} \mathrm{~s}^{-1} \mathrm{Mpc}^{-1}$. This gives a scale of $0.985 \mathrm{kpc} /{ }^{\prime \prime}$ at the redshift of the merging system, which is $z=0.05043$.

\section{THE TARGET}

The target, P96949 (RA:11:54:10.27, DEC:+00:08:19.3), is drawn from the Millennium Galaxy Catalog (Liske et al. 2003; Driver et al. 2005) and selected by Poggianti et al. (2016) from the Padova Millennium Galaxy and Group Catalogue (PM2GC, Calvi et al. 2011).

Figure 1 shows a color composite image obtained combining the reconstructed $g, r$ and $i$ filters from the MUSE datacube. The irregular shape of the galaxy immediately stands out. Two distinct components are visible: a bright, circular one dominates on the West side of the galaxy, while a more diffuse, fainter one is centered 5." 3 North-East from the other one. These two components most likely correspond to two merging galaxies, but only the integral field spectroscopy can reveal whether they are physically associated. A very bright knot in between the components already stands out for its peculiar colors.

P96949 is part of a small group of three objects. Tempel et al. (2012) computed a velocity dispersion $\sigma=60 \mathrm{~km}$ $\mathrm{s}^{-1}$. The other two group members are at 9.'2 and 12.'70 to-

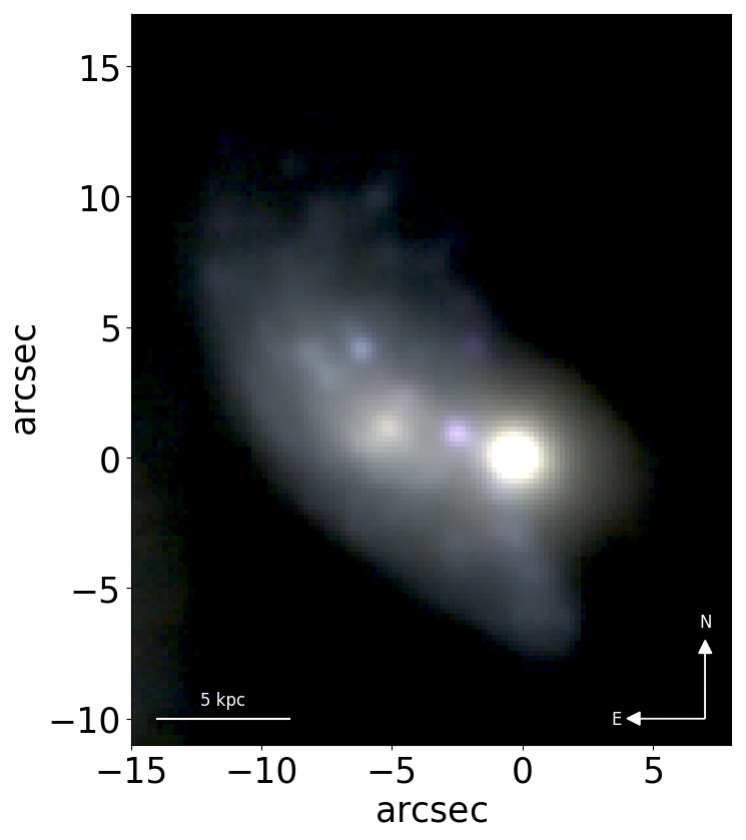

Figure 1. RGB image of P96949. The reconstructed $g, r, i$ filters from the MUSE cube have been used. North is up, and east is left. In this and all plots $(0,0)$ corresponds to the peak of the continuum underlying $\mathrm{H} \alpha$ flux (see Fig. 3).

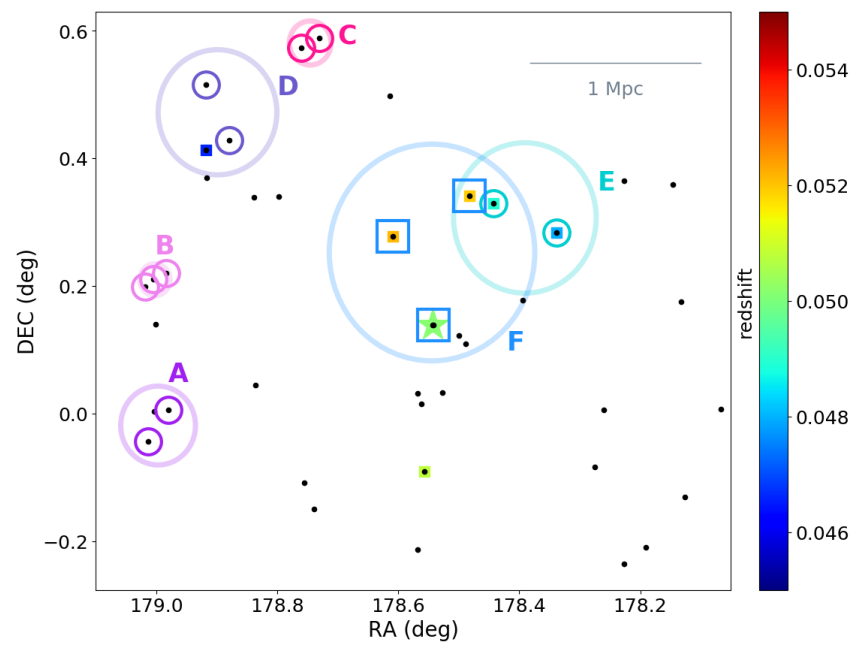

Figure 2. Position on the sky of galaxies around P96949, indicated with a green star, in the redshift range $0.01<z<0.1$ (black dots). Galaxies in the redshift range $0.045<z<0.055$ are color-coded according to their redshift (squares). Galaxies belonging to the group of P96949 (group F) are highlighted with open squares; galaxies belonging to other groups are highlighted with open circles (see Tempel et al. 2012 and Tab.1 for details). Shaded circles indicate the virial radius of the groups. The scale in the upper right corner shows $1 \mathrm{Mpc}$ at the redshift of P96949. 
Table 1. Properties of the groups around P96949

\begin{tabular}{rrrrrrr}
\hline IDcl Ngal & z & $\begin{array}{r}\text { RAJ2000 } \\
(J 2000)\end{array}$ & $\begin{array}{r}\text { DEJ2000 } \\
(\mathrm{J} 2000)\end{array}$ & $\begin{array}{c}\text { Rvir } \\
(\mathrm{Mpc})\end{array}$ & $\begin{array}{c}\sigma \\
\left(\mathrm{km} \mathrm{s}^{-1}\right)\end{array}$ \\
\hline A & 2 & 0.07853 & 178.99687 & -0.01874 & 0.224 & 38.5 \\
B & 3 & 0.07944 & 179.00206 & 0.20978 & 0.091 & 248.3 \\
C & 2 & 0.07978 & 178.74551 & 0.58081 & 0.127 & 135.6 \\
D & 2 & 0.06184 & 178.89868 & 0.47222 & 0.285 & 9.8 \\
E & 2 & 0.04844 & 178.39067 & 0.30664 & 0.273 & 198.7 \\
F & 3 & 0.0519 & 178.54411 & 0.25248 & 0.420 & 60.0 \\
\hline
\end{tabular}

NotE-Data taken from Tempel et al. (2012).

wards North. Figure 2 shows the spatial distribution of all galaxies around $\mathrm{P} 96949$ with measured redshift in the range $0.01<z<0.1$. Redshifts are taken from the MGCz (Driver et al. 2005) and SDSS-DR9 (Ahn et al. 2012). Tempel et al. (2012) identified five other groups in this area, whose position is highlighted in Fig. 2 and whose properties are listed in Tab.1. The relative positions, velocity dispersions and redshift differences between all these groups and those of that of P96949 (group "F") suggest that group-group interactions are hardly able to affect the group members.

\section{DATA}

\subsection{Observations and data reduction}

Following the GASP strategy, P96949 was observed in service mode with the MUSE spectrograph, mounted at the Nasmyth focus of the UT4 VLT, at Cerro Paranal in Chile. It was observed on $21 / 02 / 2017$, with photometric conditions; the seeing at $650 \mathrm{~nm}$ (measured on telescope guide star) remained below $0 .{ }^{\prime \prime} 9$ during the whole observing block. A total of four 675 seconds exposures were taken with the Wide Field Mode.

The data reduction process for all galaxies in the GASP survey is presented in Paper I.

\subsection{Data analysis}

The procedures used to analyze all galaxies of the GASP survey are extensively presented in Paper I. We corrected the reduced datacube for extinction due to our Galaxy. We assumed the extinction law from Cardelli et al. (1989) and used the extinction value computed at the galaxy position (Schlafly \& Finkbeiner 2011).

We analyzed the most prominent emission lines in the spectrum by exploiting the KUBEVIZ (Fossati et al. 2016) code, which yields total fluxes and the kinematic properties of the gas. As first step, we average filtered the datacube in the spatial direction with a $5 \times 5$ pixel kernel, corresponding to $1^{\prime \prime} \sim 0.99 \mathrm{kpc}$ at the galaxy redshift (see Paper I, for details). In the MUSE wavelength range the typical spectral dispersion of $1.25 \AA$ pixel $^{-1}$ translates to a velocity scale of $25 \mathrm{~km} \mathrm{~s}^{-1}$ pixel $^{-1}$. The average FWHM resolution is $2.51 \AA$, equivalently to $110 \mathrm{~km} \mathrm{~s}^{-1}$ (or $53 \mathrm{~km} \mathrm{~s}^{-1}$ pixel $^{-1}$ ). Details on the methods can be found in Paper I.

We derived the stellar kinematic from the analysis of the characteristics of absorption lines, using the Penalized PixelFitting (pPXF) software (Cappellari 2012), which works in Voronoi binned regions of given S/N (10 in this case; see Cappellari \& Copin 2012). We fitted observed spectra with the stellar population templates by Vazdekis et al. (2010). We further smoothed the value of the stellar radial velocity using the two-dimensional local regression techniques (LOESS) as implemented in the Python code developed by M. Cappellari. ${ }^{2}$ Details on the methods can be found in Paper I.

We obtained the spatially resolved properties of the stellar populations and the correction for underlying absorption to measure total emission line fluxes running the spectral fitting code SINOPSIS (Fritz et al. 2017, Paper III). Briefly, it combines different simple stellar populations spectra to reproduce the observed equivalent widths of the most prominent absorption and emission lines, and the continuum in various bands. The code uses the latest SSP model from S. Charlot \& G. Bruzual (2017, in preparation). SINOPSIS produces a best-fit model datacube for the stellar-only component and maps of stellar mass, average star formation rate and total mass formed in four age bins, luminosity-weighted and mass-weighted stellar ages. To calculate the total mass in a given spaxel, we sum the masses in the four main age bins. Due to the code characteristics, when the spectra have a low signal-to-noise, SINOPSIS tends to include an unnecessary small percentage of old $\left(t>5.7 \times 10^{8}\right)$ stars. To be conservative, we neglect the contribution of stars older than $5.7 \times 10^{8} \mathrm{yr}$ in low $\mathrm{S} / \mathrm{N}$ spectra $(\mathrm{S} / \mathrm{N}<3)$ when their contribution to the stellar continuum luminosity is less than $3 \%$. The entire contribution of young stars, instead, is taken into account, given the fact that it is estimated from the emission lines, which are more reliable features.

We then corrected the emission-line, absorption-corrected fluxes for extinction by dust internal to the galaxy. We obtained the map of the dust extinction $\mathrm{A}_{V}$ from the absorptioncorrected Balmer decrement in each spaxel. As described in Paper I, we assumed an intrinsic $\mathrm{H} \alpha / \mathrm{H} \beta$ ratio equal to 2.86 and adopt the Cardelli et al. (1989) extinction law. The $\mathrm{A}_{V}$ map has been calculated only for spaxels where the $\mathrm{S} / \mathrm{N}$ on the $\mathrm{H} \alpha$ and $\mathrm{H} \beta$ lines is larger than 3 and the ratio of the two lines is larger than the assumed 2.86 value for the Balmer decrement.

We used the line fluxes to produce line-ratio diagnostic diagrams (BPT, Baldwin et al. 1981) that allow to investigate the

\footnotetext{
2 http://www-astro.physics.ox.ac.uk/ mxc/software
} 


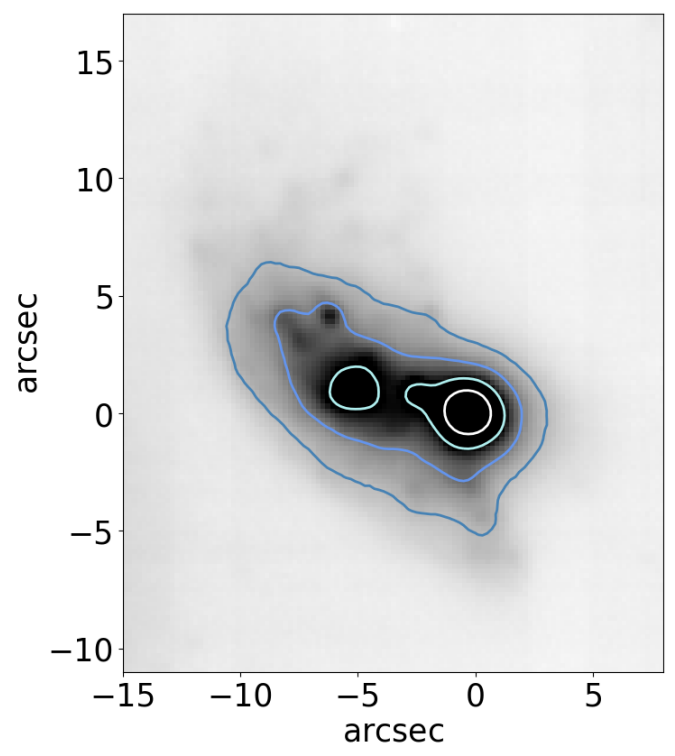

Figure 3. MUSE white-light image of P96949. Contours are logarithmically spaced isophotes of the continuum underlying $\mathrm{H} \alpha$ down to a surface brightness $2.5 \times 10^{-18} \mathrm{erg} \mathrm{s}^{-1} \mathrm{~cm}^{-2} \AA^{-1} \operatorname{arcsec}^{-2}$.

origin of the gas ionization and distinguish between regions photoionized by hot stars and regions ionized by shocks, LINERs and AGN. Only spaxels with a $S / N>3$ in all the emission lines involved are taken into account.

We calculated the gas metallicity for each star forming spaxel using the pyqz Python module7 (Dopita et al. 2013) v0.8.2; we obtained the $12+\log (O / H)$ values by interpolating from a finite set of diagnostic line ratio grids computed with the MAPPINGS code (see Paper I). As discussed in detail by Kewley \& Ellison (2008), the systematic errors introduced by modeling inaccuracies are of the order of $\sim 0.1-0.15$ dex. Instead, discrepancies among the various calibrations based on photoionization models can reach values of up to 0.2 dex.

We computed the SFR of each spatial element from the $\mathrm{H} \alpha$ luminosity corrected for dust and stellar absorption, following the Kennicutt (1998a)'s relation. We finally derived the total SFR as the sum of the dust- corrected $\mathrm{H} \alpha$ fluxes in each spaxel with a $\mathrm{S} / \mathrm{N}(\mathrm{H} \alpha)>3$.

\section{RESULTS}

Figure 3 shows the white-light image from MUSE, i.e. the image obtained integrating the light across the entire wavelength range (4750-9350 $)$ ). The irregular shape of the galaxy is well visible, along with traces of debris to the North and the few bright knots distributed across the galaxy. The stellar isophotes reveal the presence of two distinct peaks in the light distribution, indicating the presence of two components in the system.

\subsection{Spatially resolved gas properties}

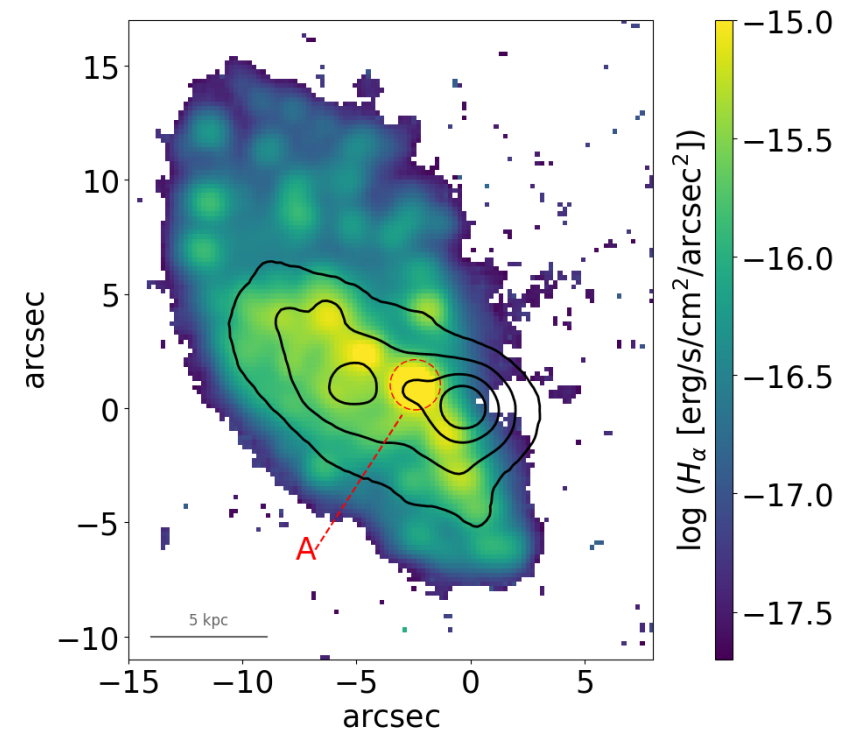

Figure 4. MUSE $\mathrm{H} \alpha$ luminosity map for spaxels with $\mathrm{H} \alpha \mathrm{S} / \mathrm{N}>4$, corrected for Galactic extinction, but uncorrected for stellar absorption and intrinsic dust extinction. Contours are continuum isophotes as in Fig.3. The position of the brightest region is highlighted in red and labeled as "A".

The image of the galaxy in the $\mathrm{H} \alpha$ light shown in Fig. 4 unveils that the gas is much more extended towards North than the stellar continuum, and less in the Western part of the galaxy. The peaks in the stellar continuum do not coincide with peaks in the $\mathrm{H} \alpha$ light. The $\mathrm{H} \alpha$ map is characterized by many bright and round $\mathrm{H} \alpha$ knots. These have different sizes depending on their spatial location: those outside of the stellar contours are smaller, the few in the center of the galaxy are larger. A particularly bright and large knot stands out at the position $\left(x=-2.5^{\prime \prime}, y=1^{\prime \prime}\right)$. Given its peculiarity, in what follows we will pay particular attention to the properties of gas and stars in that region, which we refer as "knot A".

As mentioned in Sec.3.2, the $\mathrm{H} \alpha$ fluxes presented in Fig. 4 are corrected for extinction by dust internal to the galaxy. The $A_{V}$ map (Fig. 5) shows that the dust is distributed across P96949 in a non-homogeneous way: in the South-West region the extinction is very low $\left(A_{V}<0.5 \mathrm{mag}\right)$, while the Central-East and Northern sides are dustier $\left(A_{V} \sim 1.2 \mathrm{mag}\right)$. Very dusty regions $\left(A_{V} \sim 1.4 \mathrm{mag}\right)$ are located at the positions $\left(x=-5^{\prime \prime}, y=3^{\prime \prime}\right)$ and in correspondence of knot A.

The map of $\mathrm{H} \alpha$, along with those of $\mathrm{H} \beta$, [OIII] $5007 \AA$, [OI] $6300 \AA, \mathrm{H} \alpha$, [NII] $6583 \AA$, and [SII] 6716+6731 , is used to determine the main ionizing source at each position. All the diagnostic diagrams used are concordant in finding that young stars produce the ionized gas and in excluding the presence of AGN in the galaxy center. This is in agreement with previous classifications found in the literature for the same galaxy (e.g., Véron-Cetty \& Véron 2010). 


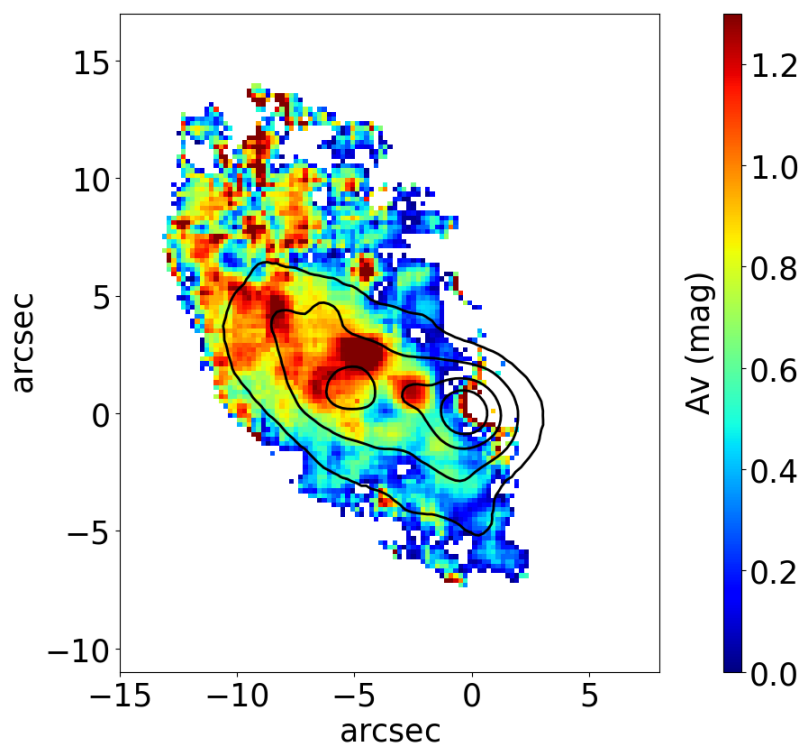

Figure 5. $A_{V}$ map. Only spaxels with a $\mathrm{S} / \mathrm{N}(\mathrm{H} \alpha)>3$ are shown. Contours are continuum isophotes as in Fig.3.



Figure 6. Metallicity map from the ionized gas. Contours are continuum isophotes as in Fig.3.

The gas metallicity of the ionized gas is shown in Fig. 6. The distribution is characterized by a quite strong gradient, varying over almost $1 \mathrm{dex}$ in $12+\log [O / H]$, with the highest metallicity regions located at the position of the nucleus of the easternmost component. This does not correspond to a peak in neither $\mathrm{H} \alpha$ nor $A_{V}$ (see Figures 4 ans 5, respectively), but it does correspond to the center of the fainter galaxy, as traced by the stellar continuum. A secondary peak is observed very close to the primary, in the position $x=-5^{\prime \prime}$, $y=3^{\prime \prime}$, where instead a star-forming region of particularly high $\mathrm{H} \alpha$ brightness and with rather high dust extinction is identified. Knot A is also characterized by relatively high values of metallicity.

\subsection{Gas and stellar kinematics}

Figure 7 shows the velocity field of the gas component. Assuming as zero point of the velocity the redshift of the galaxy, the gas rotates along the South-East/North-West direction and spans the velocity range $-50<v\left(\mathrm{~km} \mathrm{~s}^{-1}\right)<80$. The median error on the velocities is $1.4 \mathrm{~km} \mathrm{~s}^{-1}$. The gas presents a regular rotation in the outskirts, with positive velocity values in the Northern region, negative velocity values in the Southern one. In the center, the locus of the zero velocity is highly distorted and forms a " $y$ " shape. The gas velocity dispersion is overall quite low ( $\sigma_{\text {gas }}=10-20 \mathrm{~km} \mathrm{~s}^{-1}$, with a median error of $3.5 \mathrm{~km} \mathrm{~s}^{-1}$ ), indicative of a dynamically cold medium, except at the position of the knot A, where it reaches peaks of $\sim 50 \mathrm{~km} \mathrm{~s}^{-1}$. Similar values are found at the edges of P96949, but hey are probably due to the low S/N ratio of these regions.

The left panel of Figure 8 shows the velocity field of the stellar component. We choose as zero point of the velocity the same of that of the gas. Two distinct constituents are visible: the East side of the galaxy presents low stellar velocities, ranging from $\sim-80 \mathrm{~km} \mathrm{~s}^{-1}$ to $\sim 80 \mathrm{~km} \mathrm{~s}^{-1}$. In this region stars and gas have similar velocities. In contrast, the West side of the galaxy presents much higher velocities, in the range $200<v\left(\mathrm{~km} \mathrm{~s}^{-1}\right)<350$. The median error on the velocities is $36 \mathrm{~km} \mathrm{~s}^{-1}$. The relative velocity of the two sides of the system is $\sim 200 \mathrm{~km} \mathrm{~s}^{-1}$, suggesting that they are indeed physically associated.

The velocity dispersion map shown in the right panel of Figure 8 is very chaotic and regular trends are not detectable. The North-East side generally shows lower $\sigma_{\text {star }}$ values $\left(\sim 30 \mathrm{~km} \mathrm{~s}^{-1}\right)$, while the South-West side is characterized by much higher values (up to $\sim 250 \mathrm{~km} \mathrm{~s}^{-1}$ ). The median error on the velocity dispersions is $57 \mathrm{~km} \mathrm{~s}^{-1}$.

\subsection{Spatially resolved stellar properties}

The distribution of the stellar luminosity weighted age presented in Fig. 9 follows a similar spatial pattern as the one depicted in the stellar kinematics. The region Western to the $(x \sim 0)$ position has a typical age of $5 \times 10^{9} \mathrm{yr}$, while the rest of the galaxy is characterized by much younger ages, of $5 \times 10^{8}$ yr. The knot A has a even younger age, $\sim 10^{7} \mathrm{yr}$. The median relative error on the luminosity weighted ages is of the order of $15 \%$.

The stellar mass density shown in the left panel of Fig. 10 is characterized by a double peak, roughly corresponding to the peaks of the stellar continuum. The brighter peak has a stellar mass density as high as $5 \times 10^{9} \mathrm{M}_{\odot} / \mathrm{kpc}^{2}$, while the secondary peak, which is also more spread, reaches values of $5 \times 10^{8} \mathrm{M}_{\odot} / \mathrm{kpc}^{2}$. The Northern part of the galaxy carries 

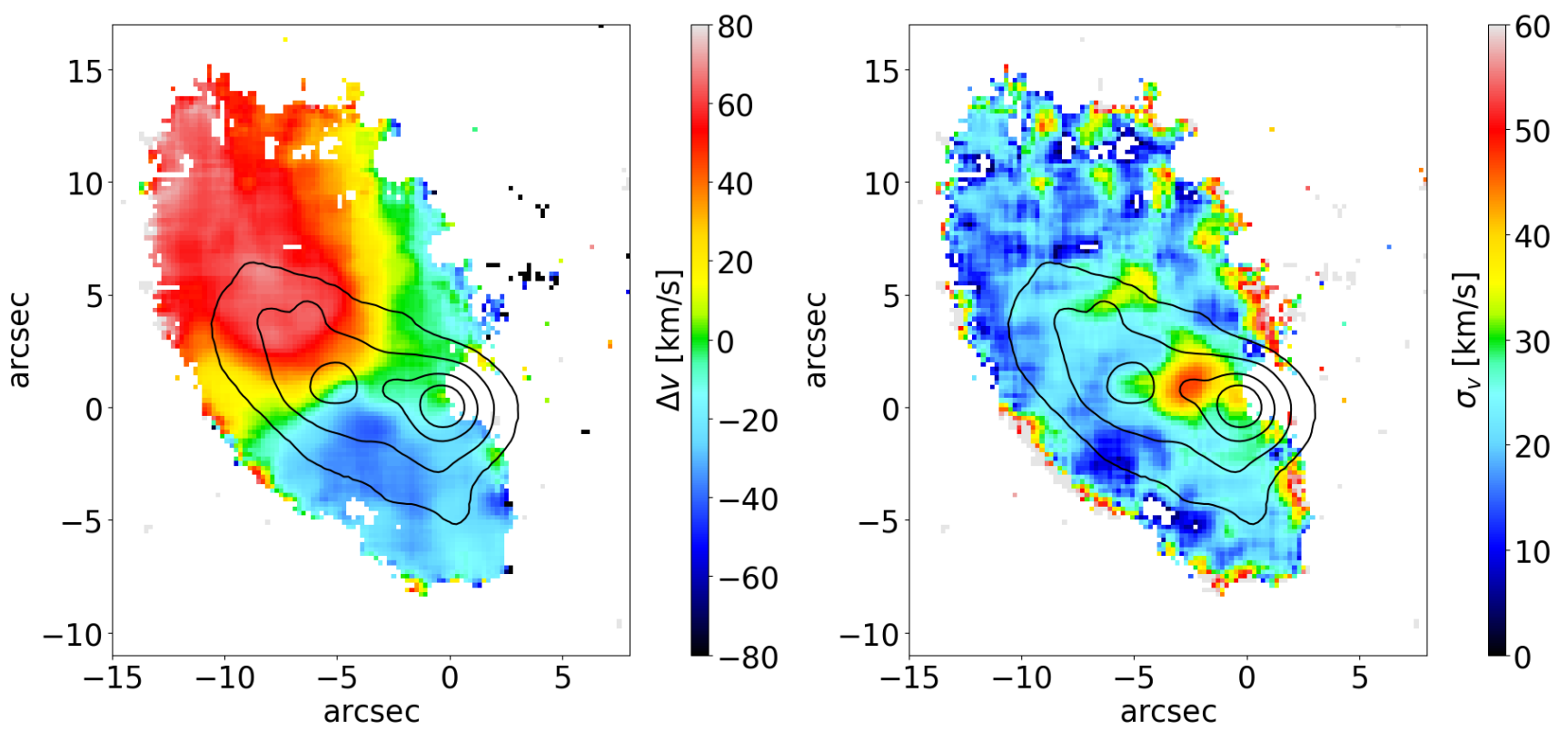

Figure 7. Gas kinematics maps for $5 \times 5$ spaxels with $\mathrm{S} / \mathrm{N} \mathrm{H} \alpha>4$. Left: $\mathrm{H} \alpha$ velocity. Right: $\mathrm{H} \alpha$ velocity dispersion. Contours are continuum isophotes as in Fig.3.
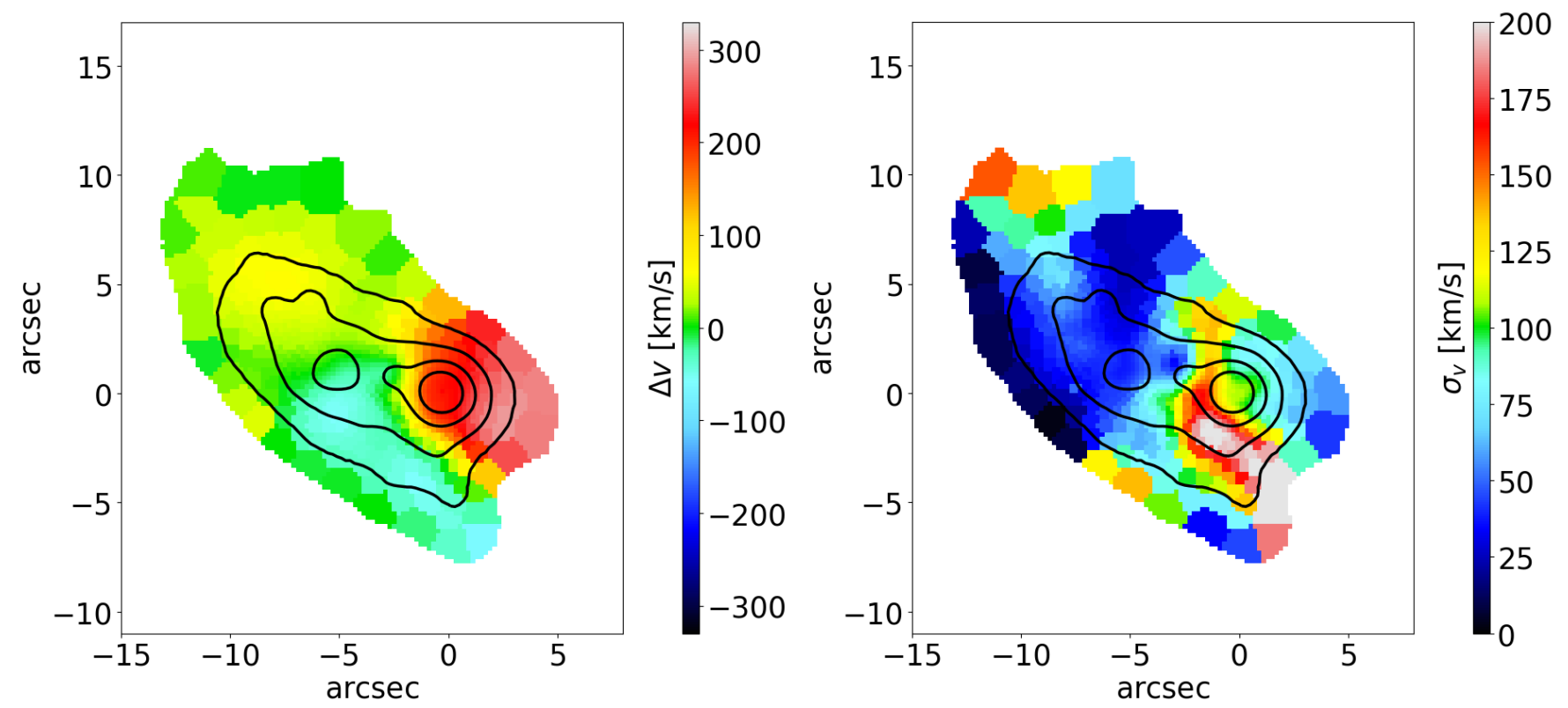

Figure 8. Stellar kinematics maps for Voronoi bins with $\mathrm{S} / \mathrm{N}>10$. Left: velocity. Right: velocity dispersion. Contours are continuum isophotes as in Fig.3.

much less mass: its typical surface mass density is $\sim 5 \times$ $10^{7} \mathrm{M}_{\odot} / \mathrm{kpc}^{2}$. Running SINOPSIS on the integrated spectra of the entire galaxy, we obtain a total $\mathrm{M}_{*}$ of $2.5 \times 10^{10} \mathrm{M}_{\odot}$,

The mass density does not trace the distribution of the recent star formation, shown in the right panel of Fig. 10. The median error on the logarithm of SFRD, computed propagating the error on the $\mathrm{H} \alpha$ flux as computed by KUBEVIZ, is 0.01 dex. Indeed, there is almost no star formation at the position of the densest component. The peak of the star formation is taking place in the knot A. An arc-shape trail of highly star forming regions centered at this position extends both toward South-West and North-East. Star forming regions are also evident on the northernmost edge of the galaxy. Integrating the spectrum over all the spaxels with $\mathrm{S} / \mathrm{N}(\mathrm{H} \alpha)>4$, we get a value of $\mathrm{SFR}=1.8 \mathrm{M}_{\odot} \mathrm{yr}^{-1}$.

Figure 11 shows the spatially resolved star formation history. This map allows us to characterize how many stars were formed at each spatial location as a function of cosmic time. 


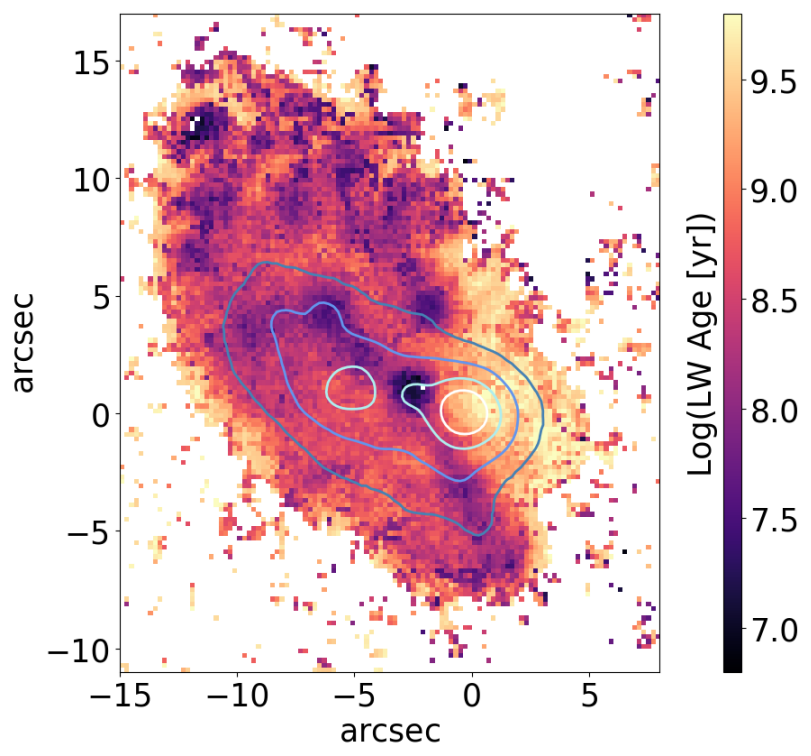

Figure 9. Luminosity weighted age (LWA) map. Contours are continuum isophotes as in Fig.3.

We choose four logarithmically spaced age bins in such a way that the differences between the spectral characteristics of the stellar populations are maximal (Fritz et al. 2007 and Paper III). The median errors on the SFR per $\mathrm{kpc}^{2}$ are of the order of $0.003 M_{\odot} y r^{-1} k_{p c}^{-2}$ in the two youngest age bins, of the order of $0.007 M_{\odot} y r^{-1} k p c^{-2}$ in the two oldest age bins. The upper left panel shows that the ongoing SFR $\left(t<2 \times 10^{7}\right.$ $\mathrm{yr}$ ) is very intense in the central region of the system, but is essentially absent in the Westernmost side where there is no ionized gas (Fig.4). High star formation activity is observed in the knot A, while low-levels of star formation are detected in the Northern part of the galaxy. The recent star formation activity $\left(2 \times 10^{7} \mathrm{yr}<t<5.7 \times 10^{8} \mathrm{yr}\right.$, upper right panel) has a similar spatial distribution compared to the youngest stars, even though recent star formation extends towards the western part of the galaxy, while the northern part is less extended. At these ages, the knot $\mathrm{A}$ is not visible anymore.

As we move to the intermediate $\left(5.7 \times 10^{8} \mathrm{yr}<t<5.7 \times\right.$ $10^{9} \mathrm{yr}$, bottom left panel) and old $\left(t>5.7 \times 10^{9} \mathrm{yr}\right.$, bottom right panel) populations, the distribution of the stars is different, being mostly confined within the continuum isophotes. All the westernmost region of the galaxy was star forming at those epochs, as was the brightest of the two components of the system.

\subsection{Properties of the two merging components}

To characterize the morphology of the two objects of the analyzed system, we use the white-light image shown in Fig. 3 to model the two components, using an iterative method to separate their contribution to the total light. In particular, the purposely devised software AIAP (by Giovanni Fasano, private communication) allows us to perform adap- tive smoothing, interactive masking and ellipse fitting of each galaxy isophote, as well as to generate a smooth model faithfully reproducing the shape of the real galaxies, including position angle and ellipticity profiles. Such an accurate modeling procedure has been iteratively applied to the early-typelike (first step) and late-type components of the system, each time removing the model from the original image. The end products of this iteration are the final model of the early-typelike galaxy and the residual image of the late-type galaxy (see Fig. 12).

Fitting the early-type component with a Sersic law, we obtian a value for the Sersic index $n$ of 2.51 and a value for the effective radius $R_{e}$ of $1.8 \mathrm{kpc}$. The total luminosity in the wavelength range $4750-9350 \AA$ is $2.2 \times 10^{19} \mathrm{erg} / \mathrm{s}$. The parameters of the late-component are instead $n=0.2, R_{e}=5.3$ $\mathrm{kpc}$, total luminosity $=2.9 \times 10^{19} \mathrm{erg} / \mathrm{s}$.

The median LWA for the early-type galaxy is $\sim 2 \times 10^{9} \mathrm{yr}$, that for the late-type galaxy is instead significantly lower: $\sim 2.5 \times 10^{8} \mathrm{yr}$.

Running SINOPSIS on the integrated spectra of the early type (obtained from the aforementioned model), we obtain a $M_{*}$ of $1.4 \times 10^{10} \mathrm{M}_{\odot}$. Subtracting this value to the total stellar mass of the system, we infer a stellar mass for the late-type component equal to $\sim 1.1 \times 10^{10} \mathrm{M}_{\odot}$. These values have to be taken with caution, given the physical spatial overlap of the two galaxies, with the consequence that a number of spaxels contribute to both components.

\section{DISCUSSION}

In the previous section we have described the most salient properties of P96949. Here we connect all of them to draw a scenario able to explain the formation and evolution of the galaxy.

P96949 is part of a small group with three members. Nonetheless, we have not identified any sign of disturbance due to the presence of the other two galaxies, which are located at a projected distance of $540 \mathrm{kpc}$ and $750 \mathrm{kpc}$, respectively.

Our interpretation is that we are witnessing an ongoing $\sim 1: 1$ merger between two galaxies with distinct properties.

A gas-poor early-type galaxy has probably recently merged with a late-type one. The relative motion of the stars of the two components is $\sim 200 \mathrm{~km} \mathrm{~s}^{-1}$, indicating that the two systems are indeed merging. Currently, the early-type galaxy has a higher line-of-sight velocity. The gas motion is disturbed on the side of the collision.

The absence of prominent tidal tails, which usually form after the first passage (e.g. Mihos \& Hernquist 1996), in the late-type indicates that the early-type is in its first approach. Note, however, that tails often fade away at large distances due to surface brightness dimming (e.g. Hibbard \& Vacca 1997; Overzier et al. 2010; Hung et al. 2014). 

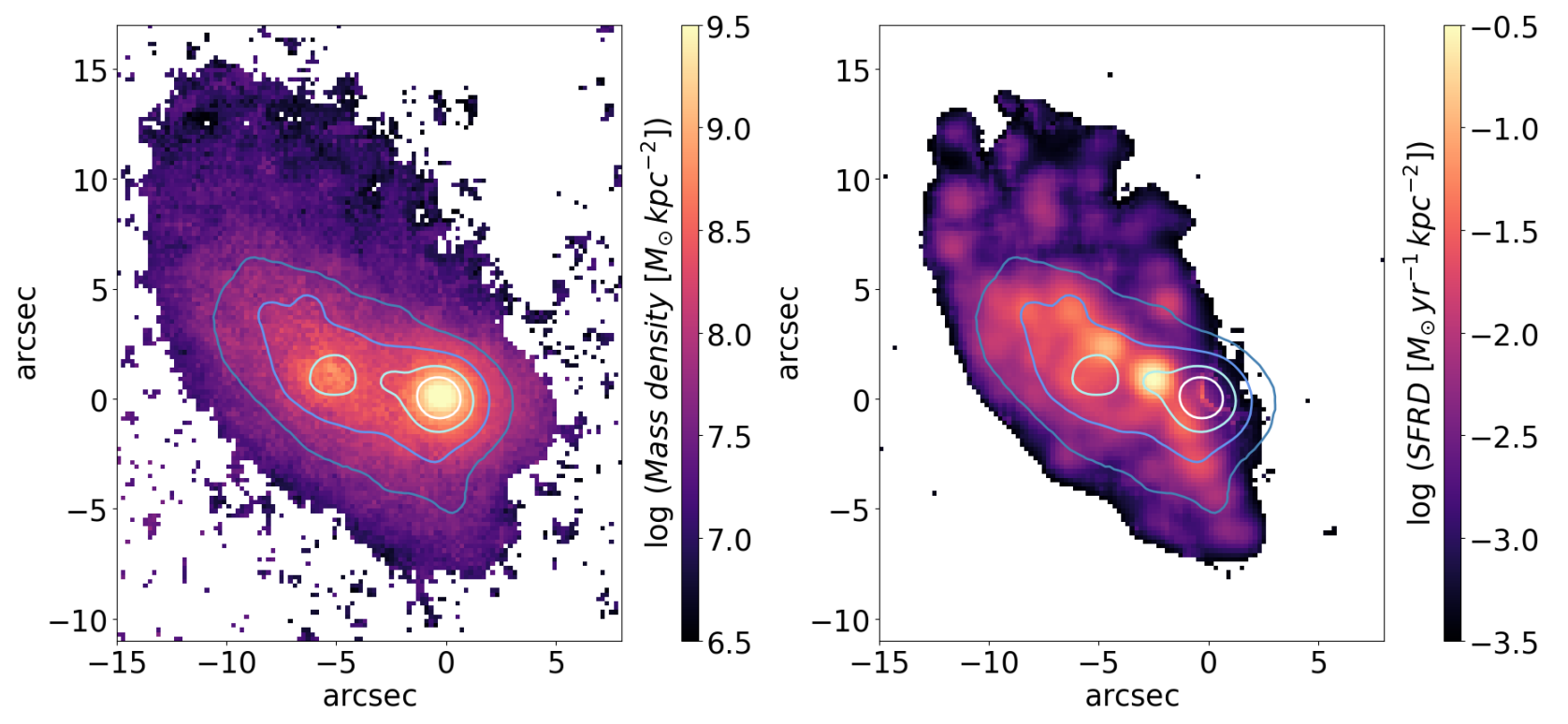

Figure 10. Left: Stellar mass density map. Right: Star formation density map. In both panels, contours are continuum isophotes as in Fig.3.

The early-stage of the merger is also corroborated by the rather strong metallicity gradient we have observed in the system. Indeed, models of inside-out disk growth predict that gradients should be initially steep and become flatter at later times (e.g. Prantzos \& Boissier 2000; Magrini et al. 2007; Fu et al. 2009; Marcon-Uchida et al. 2010), after the gas inflows of relatively metal-poor gas has had the time to change the galaxy's metal distribution (Mihos \& Hernquist 1996; Barnes \& Hernquist 1996). N-body/SPH numerical simulations of equal-mass mergers by Rupke et al. (2010) also show that between first and second pericenter the initial radial metallicity profile noticeably flattens. This smoothing reflects the effects of gas redistribution over the galaxy disk.

During the interaction, there has been an enhancement of the star formation activity in the central part of the system. Moreno et al. (2015), employing a suite of 75 SPH merger simulations, have found that whenever enhanced star formation in the nucleus is triggered, this is always accompanied by the suppression of the star formation activity at large galactocentric radii, similarly to what we observe in P96949.

The high star formation gives rise to enhancements in the metallicity of the central part of the galaxy, in agreement to predictions of N-body/smooth particle hydrodynamics models (Torrey et al. 2012).

The overall gas redistribution does not excessively boost the star formation: P96949 lays on the typical SFR-mass relation for star-forming field galaxies (see Poggianti et al. 2016). This is true both if we consider the total stellar mass and if we assume that all the star formation comes from the late-type galaxy.

We can try to date the merging event comparing the stellar maps of different ages, as done in Fig.13. The contours representing the populations with $t>5.7 \times 10^{9} \mathrm{yr}$ highlight the presence of two distinct peaks in the star formation distribution, with less stars forming between them. On the contrary, the populations with $t<5.7 \times 10^{8} y r$ highlight the presence of new born material in between the two components, suggesting that the interaction has started $2 \times 10^{7} \mathrm{yr}<\mathrm{t}<5.7 \times 10^{8} \mathrm{yr}$ ago and is still ongoing. The forming stellar structure in the central part of the merging system corresponds to the region that we have called knot A. Approximately at the same time, the merger has been also moving gas towards the Norhtern part of the galaxy, where new-born stars are observed. New high resolution simulations (Teyssier et al. 2010; Hopkins et al. 2013; Renaud et al. 2015) show that during the first two pericenter passages extended star formation arises spontaneously. This is most likely due to fragmentation of the gas clouds produced by an increase of the interstellar medium supersonic turbulence as a consequence of the tidal interaction itself (Renaud et al. 2014).

The build up of new stars produces a lopsided morphology to the resulting galaxy. As also shown in Fig.11, the gas rich object had a regular shape at the early stages of its formation, while for $t<5.7 \times 10^{8} y r$ it started developing an asymmetric shape.

Lopsidedness induced by tidal encounter between two galaxies with an arbitrary orientation is a common feature in group galaxies (e.g. Combes et al. 2004), where the perturbation is expected to generate a force term, responsible for the lopsidedness in the galaxy. Lopsidedness can also be generated due to a mild interaction or more indirectly due to the response of the disk to the distorted halo which feels a stronger effect of the interaction (Weinberg 1995; Jog 1997; Schoenmakers et al. 1997).

\subsection{The formation of a Tidal Dwarf Galaxy}




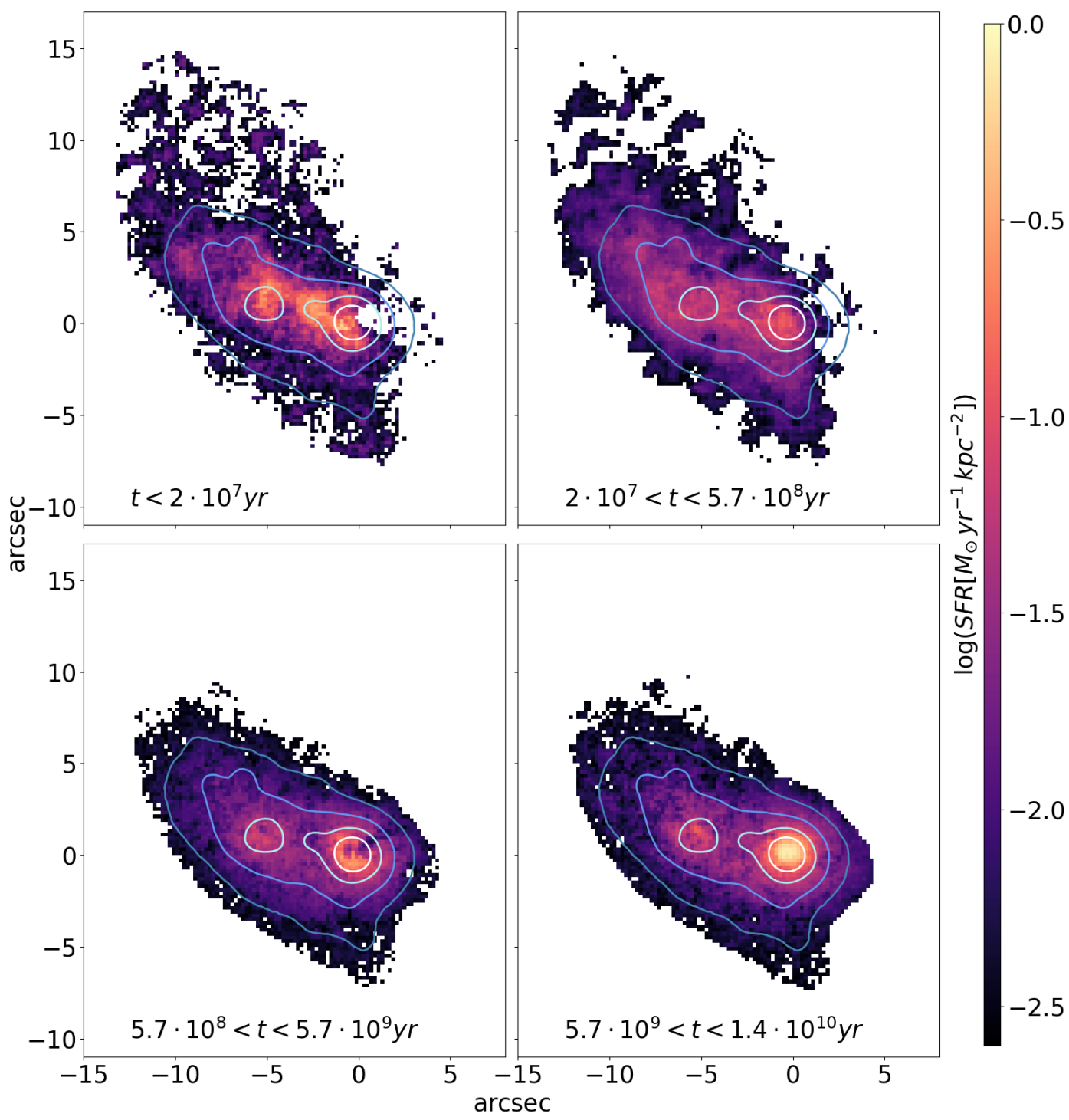

Figure 11. Average star formation rate per $\mathrm{kpc}^{2}$ in four different ages: during the last $2 \times 10^{7} \mathrm{yr}$ (top left), between $2 \times 10^{7} \mathrm{yr}$ and $5.7 \times 10^{8} \mathrm{yr}$ (top right), $5.7 \times 10^{8} \mathrm{yr}$ and $5.7 \times 10^{9} \mathrm{yr}$ (bottom left) and $>5.7 \times 10^{9} \mathrm{yr}$ ago (bottom right). Contours are continuum isophotes as in Fig.3.

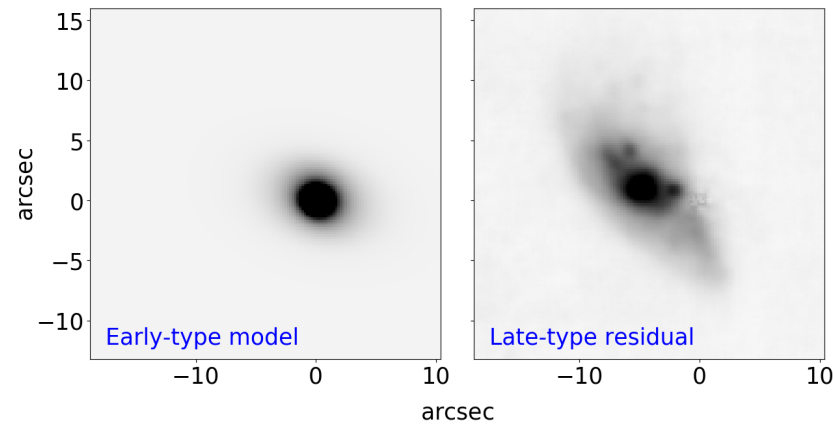

Figure 12. Left. Model for the early-type component based on the MUSE white-light image. Right. Residual image for the late-type galaxy. See text for details.
As mentioned in the previous section, the merger between the two galaxies induces the formation of a number of stellar structures. The conditions in which the structures form determine whether it is most likely SSCs or TDGs (see Sect.1).

According to Duc (2012), TDGs should mostly be produced by major wet mergers that occurred less than one Gyr ago with low impact velocities (up to $250 \mathrm{~km} \mathrm{~s}^{-1}$ ) involving at least one spiral galaxy and are formed by material that used to belong to the parent galaxies. The production rate is about 1 TDG per favorable merger (see also Bournaud et al. 2007). Being recycled objects, TDGs have inherited the metal content of the interstellar medium from their progenitors. Thus, their metallicity gives information about the past chemical enrichment of the progenitors, and is not correlated with their current mass, contrary to normal galaxies. TDGs 




Figure 13. Comparison between the stellar maps of different ages. The ongoing star formation bin $\left(t<2 \times 10^{7} \mathrm{yr}\right.$, black and gray lines) is shown in all the panels, for reference. Left: recent star formation bin $\left(2 \times 10^{7} \mathrm{yr}<\mathrm{t}<5.7 \times 10^{8} \mathrm{yr}\right.$, blue lines $)$. Middle: intermediate star formation bin $\left(5.7 \times 10^{8} \mathrm{yr}<\mathrm{t}<5.7 \times 10^{9} \mathrm{yr}\right.$, green lines $)$. Right: old star formation bin $\left(t>5.7 \times 10^{9} \mathrm{yr}\right.$, red lines). Contours are logarithmically spaced between $\mathrm{SFR}=0.0001$ and $0.1 M_{\odot} y r^{-1}$.

have masses around $10^{8} M_{\odot}$, and are young objects. They still exhibit the tails and bridges in which they were formed that have not had the time to evaporate yet. Once evolved, TDGs should become indistinguishable from regular satellite galaxies on optical images.

According to simulations (Duc et al. 2004; Bournaud \& Duc 2006; Wetzstein et al. 2007), TDGs should also be free of dark matter. Indeed, their low escape velocity is low (at most a few tens of $\mathrm{km} \mathrm{s}^{-1}$ ), therefore the dark matter particles of the halo of the parent spiral galaxy will not be held by the gravitational well of the TDG.

Since TDGs are made-up from material coming from the disk of the progenitor, the dynamical mass measured from their rotation velocity and size should be similar to their visible mass in stars and gas (Zackrisson \& Riehm 2010; Bournaud 2010).

In our analysis we have have paid particular attention to a structure formed from the impact between the two galaxies, located at $\left(x=-2.5^{\prime \prime}, y=1^{\prime \prime}\right)$ that we called knot A.

The location and radius of the knot are found through a purposely devised script developed in the context of the GASP survey (see Paper I). The knot's radius is estimated through a recursive analysis, taking care of deblending the contributions from overlapping sources and is $\sim 2.2 \mathrm{kpc}$. This value is well above the seeing $\left(\sim 1^{\prime \prime}\right)$ and the MUSE PSF.

We then run KUBEVIZ and SINOPSIS on a mask identifying only the knot, to obtain its properties. As the analysis of emission-line ratios shows that star formation is the main source of ionization, we can also compute the electron density $n_{e}$ and the mass of the ionized gas $M_{\text {gas }}$, following the relation presented in Proxauf et al. (2014) and described in detail in Paper I.

We estimate the following quantities for the knot A: $A_{V}=0.85 \mathrm{mag}, 12+\log (O / H)=8.4, n_{e}=54 \mathrm{~cm}^{-3}, M_{\text {gas }}=$ $2.5 \times 10^{6} M_{\odot}, M_{\text {star }}=6.3 \times 10^{9} M_{\odot}$, mass density $=4.1 \times$ $10^{8} M_{\odot} k p c^{-2}$, luminosity weighted age $=\sim 3 \times 10^{7} \mathrm{yr}$, $\mathrm{SFR}=0.3 M_{\odot} y r^{-1}, \mathrm{SFRD}=0.02 M_{\odot} y r^{-1} k p c^{-2}$.

This knot therefore is very massive and large, has one of the largest metallicity values of the system, and is relatively young. It has also a high SFR and it is relatively dusty. It was born in correspondence of the merger, and connection with the parent galaxy is still visible and is most likely a spiral arm. Its dynamical mass is $M_{d y n}=2 \times r \frac{\sigma_{r}^{2}}{G} \sim 2.5 \times 10^{9} M_{\odot}$ if we adopt $R_{e} \sim 2.2 \mathrm{kpc}$ and $\sigma_{r} \sim 50 \mathrm{~km} \mathrm{~s}^{-1}$. This value is very similar to its stellar mass, suggesting that there is not dark matter in the structure.

This object is therefore a good candidate for being or soon becoming a TDG. Nonetheless, it presents peculiar properties.

Kaviraj et al. (2012) have conducted a statistical observational study of the TDGs in the nearby Universe. They found that $95 \%$ of TDG-producing mergers involve interactions between two blue, spiral galaxies. The vast majority of these parent systems have mass ratios greater than $\sim 1: 7$. Only a fifth of gas-rich major mergers produce TDGs with masses 
greater than $10^{8} M_{\odot}$. TDGs are usually within $\sim 20 \mathrm{kpc}$ of their parent galaxies.

Differently from other TDG candidates (see also Sengupta et al. 2014), knot A has formed in a very early stage of the merger and even though it is still in the phase of forming and still located within the disk of its progenitor, it has already been able to accrete great quantities of gas and stars and has high and unusual SFR values.

Another explanation might be that instead of being a merger-induced TDG, the observed structure is a huge (up to a few $10^{8} M_{\odot}$ ) star-forming region, similarly to what detected at similar redshift by e.g. Fisher et al. (2014) and at higher redshift $(1<z<3)$ by e.g. Genzel et al. (2011); Elmegreen \& Elmegreen (2005); Elmegreen et al. (2009). However, this interpretation requires disk-dominated systems and high gas fractions ( $>30-50 \%)$, which are not the case for P96949. We can roughly estimate the gas fractions from the stellar mass and star formation density maps shown in Fig.10 and the Kennicutt (1998b) law. We get a gas fraction of $\sim 10-15 \%$ if the system is on the low-efficiency side on the relation, and even less if the system is on the higher side, as expected for a merger (Daddi et al. 2010). As a consequence, both the TDG and the other structures detected as bright blue regions in formation (Fig.1) are most likely merger-induced and not simply large SF regions intrinsic to this particular system.

A third option might be that knot A is a tidally induced star forming region that was triggered by the tidal/compressive forces of the merger. This possibility would not require large disk gas fractions. If the structure will remain kinematically bound to the parent galaxy, it will not turn into a TDG. As we are observing knot $\mathrm{A}$ in an early stage of its life, we can not firmly state that it will certainly become a long-lived TDG. However, its peculiar properties, and in particular its very high velocity dispersion makes it a remarkable object and a very good TDG candidate, rather than simply a massive tidally-induced star forming region within a merging system.

To conclude, we note that knot A seems to be in a filament of star formation regions triggered by the merger. Indeed, any merger is expected to create a number of starforming knots, with a range of masses. However, simulations show that only the most massive ones tend to survive (see, e.g. Bournaud \& Duc 2006). We can identify two other structures with quite peculiar characteristics in the filament. These are located at $\left(x=-5^{\prime \prime}, y=3^{\prime \prime}\right)$, and at $\left(x=-7^{\prime \prime}\right.$, $y=5^{\prime \prime}$ ), respectively. In addition to be smaller in size (radius $\sim 1.6$ and $1.8 \mathrm{kpc}$, respectively), they are also considerably less star forming $\left(\mathrm{SFR} \sim 0.09 \mathrm{M}_{\odot} \mathrm{yr}^{-1}\right.$, SFRD $\sim 0.001$ and $\left.0.009 M_{\odot} y r^{-1} k p c^{-2}\right)$, less massive $\left(\mathrm{M}_{*} \sim 8 \times 10^{8}\right.$ and $\mathrm{M}_{*} \sim 1.4 \times 10^{9} \mathrm{M}_{\odot}$, mass density $\sim 10^{8}$ and $\mathrm{M}_{*} \sim 1.4 \times$ $10^{8} \mathrm{M}_{\odot} \mathrm{kpc}^{-2}$ ), older (luminosity weighted age $\sim 1.3 \times 10^{8}$ and $\left.\sim 1.5 \times 10^{8} y r\right)$ and are characterised by lower values of velocity dispersion $\left(\sim 25 \mathrm{~km} \mathrm{~s}^{-1}\right)$. Rather than being TDG candidates, these will therefore most likely become SSCs, or, if massive enough, eventually globular clusters (Bournaud et al. 2008).

\section{CONCLUSIONS}

GASP (GAs Stripping phenomena in galaxies with MUSE) is an ongoing ESO Large Program with the MUSE/VLT to investigate the causes and the effects of gas removal processes in galaxies in different environments. The sample includes galaxies selected for showing signs that could be indicative of stripping in B-band images.

Within the sample, we identified an ongoing merger between two galaxies with different properties. This peculiar system is so far unique in the GASP sample, suggesting that usually mergers are not confused with galaxies undergoing gas stripping.

A gas poor, early-type-like object $\left(n=2.51, R_{e}=1.8 \mathrm{kpc}\right.$, $M_{*}=1.4 \times 10^{10} M_{\odot}$ ) with a luminosity weighted age of $\sim 2 \times$ $10^{9} \mathrm{yr}$ has started an interaction with a younger (luminosity weighted age $\sim 2.5 \times 10^{8} \mathrm{yr}$ ), gas rich, late-type object ( $n=$ $0.2, R_{e}=5.3 \mathrm{kpc}, M_{*}=1.1 \times 10^{10} M_{\odot}$ ) between $2 \times 10^{7} \mathrm{yr}<$ $\mathrm{t}<5.7 \times 10^{8} \mathrm{yr}$ ago. The merger is still in an early phase. The gas kinematic pattern reflects the gas of the late-type object and is mainly distorted in correspondence to the location of the impact, while the northern regions had not time yet to be influenced. The stellar kinematic instead is much more chaotic, as expected in case of mergers. Nonetheless, the rotation of the stars in the two galaxies is still detectable. The early-type galaxy has currently a higher line-of-sight velocity than the late-type galaxy.

The merger induces a gas redistribution igniting high levels of star formation both in the northern part of the galaxy, producing a lopsidedness visible for $t<5.7 \times 10^{8} y r$, and between the two galaxies, especially in the region of the impact. As a consequence, a large stellar structure forms. This object presents peculiar properties that make it a good TDG candidate. Indeed, it has formed in a very early stage of the merger and even though it is still in the phase of forming and still located within the disk of its progenitor, it has already accreted great quantities of gas and stars $\left(M_{*} \sim 6 \times 10^{9} M_{\odot}\right.$, $R \sim 2.2 \mathrm{kpc}$ ) and has high and unusual SFR values (SFR $\left.0.3 M_{\odot} y r^{-1}\right)$.

This is the first detailed characterization of a TDG candidate detected beyond the local Universe, where data have relatively lower resolution. It could pave the way to search for TDGs at higher redshift, a regime still poorly characterized.

We thank the referee for their useful comments that helped us to improve the manuscript. Based on observations collected at the European Organisation for Astronomical Research in the Southern Hemisphere under ESO programme 196.B-0578. This work made use of the KUBEVIZ software 
which is publicly available at http://www. mpe.mpg . de/ dwilman/kubeviz/. We are grateful to Joe Liske, Simon Driver and the whole MGC collaboration for making their dataset easily available, and to Rosa Calvi for her valuable work on the PM2GC. We also thank Fredric Bournaud for helpful comments on a draft of this paper. We acknowledge financial support from PRIN-INAF 2014. B.V. acknowledges the support from an Australian Research Coun- cil Discovery Early Career Researcher Award (PD0028506). J.F. acknowledges financial support from UNAM-DGAPAPAPIIT IA104015 grant, México. This work was co-funded under the Marie Curie Actions of the European Commission (FP7-COFUND).

\section{Facilities: VLT(MUSE)}

Software: KUBEVIZ, ESOREX, SINOPSIS, IRAF, CLOUDY, pyqz, IDL, Python

\section{REFERENCES}

Ahn, C. P., Alexandroff, R., Allende Prieto, C., et al. 2012, ApJS, 203, 21

Baldwin, J. A., Phillips, M. M., \& Terlevich, R. 1981, PASP, 93, 5

Barnes, J. E., \& Hernquist, L. 1996, ApJ, 471, 115

Barnes, J. E., \& Hernquist, L. E. 1991, ApJL, 370, L65

Blumenthal, G. R., Faber, S. M., Primack, J. R., \& Rees, M. J. 1984, Nature, 311, 517

Boquien, M., Duc, P.-A., Braine, J., et al. 2007, A\&A, 467, 93

Bournaud, F. 2010, in Astronomical Society of the Pacific Conference Series, Vol. 423, Galaxy Wars: Stellar Populations and Star Formation in Interacting Galaxies, ed. B. Smith, J. Higdon, S. Higdon, \& N. Bastian, 177

Bournaud, F., \& Duc, P.-A. 2006, A\&A, 456, 481

Bournaud, F., Duc, P.-A., \& Emsellem, E. 2008, MNRAS, 389, L8

Bournaud, F., Duc, P.-A., Brinks, E., et al. 2007, Science, 316, 1166

Brinchmann, J., Charlot, S., White, S. D. M., et al. 2004, MNRAS, 351,1151

Bundy, K., Bershady, M. A., Law, D. R., et al. 2015, ApJ, 798, 7

Calvi, R., Poggianti, B. M., \& Vulcani, B. 2011, MNRAS, 416, 727

Cappellari, M. 2012, pPXF: Penalized Pixel-Fitting stellar kinematics extraction, Astrophysics Source Code Library, ascl:1210.002

Cappellari, M., \& Copin, Y. 2012, Voronoi binning method, Astrophysics Source Code Library, ascl:1211.006

Cardelli, J. A., Clayton, G. C., \& Mathis, J. S. 1989, ApJ, 345, 245

Chabrier, G. 2003, PASP, 115, 763

Chapon, D., Mayer, L., \& Teyssier, R. 2013, MNRAS, 429, 3114

Combes, F. 2003, in Astronomical Society of the Pacific Conference Series, Vol. 290, Active Galactic Nuclei: From Central Engine to Host Galaxy, ed. S. Collin, F. Combes, \& I. Shlosman, 411

Combes, F., Boisse, P., Mazure, A., \& Blanchard, A. 2004

Cortijo-Ferrero, C., González Delgado, R. M., Pérez, E., et al. 2017a, MNRAS, 467, 3898

—. 2017b, ArXiv e-prints, arXiv:1707.05324

—. 2017c, ArXiv e-prints, arXiv:1706.01896

Cox, T. J., Dutta, S. N., Di Matteo, T., et al. 2006, ApJ, 650, 791

Daddi, E., Elbaz, D., Walter, F., et al. 2010, ApJL, 714, L118
Dekel, A., \& Silk, J. 1986, ApJ, 303, 39

Di Matteo, T., Springel, V., \& Hernquist, L. 2005, Nature, 433, 604

Dopita, M. A., Sutherland, R. S., Nicholls, D. C., Kewley, L. J., \& Vogt, F. P. A. 2013, ApJS, 208, 10

Driver, S. P., Liske, J., Cross, N. J. G., De Propris, R., \& Allen, P. D. 2005, MNRAS, 360, 81

Duc, P.-A. 2012, Astrophysics and Space Science Proceedings, 28, 305

Duc, P.-A., Bournaud, F., \& Masset, F. 2004, A\&A, 427, 803

Efstathiou, G., Moody, S., Peacock, J. A., et al. 2002, MNRAS, 330, L29

Elmegreen, B. G. 2011, in EAS Publications Series, Vol. 51, EAS Publications Series, ed. C. Charbonnel \& T. Montmerle, 45

Elmegreen, B. G., \& Elmegreen, D. M. 2005, ApJ, 627, 632

Elmegreen, B. G., Elmegreen, D. M., Fernandez, M. X., \& Lemonias, J. J. 2009, ApJ, 692, 12

Elmegreen, B. G., Kaufman, M., \& Thomasson, M. 1993, ApJ, 412, 90

Fensch, J., Duc, P.-A., Weilbacher, P. M., Boquien, M., \& Zackrisson, E. 2016, A\&A, 585, A79

Fernández, M., Yuan, F., Shen, S., et al. 2015, Galaxies, 3, 156

Fisher, D. B., Glazebrook, K., Bolatto, A., et al. 2014, ApJL, 790, L30

Fossati, M., Fumagalli, M., Boselli, A., et al. 2016, MNRAS, 455, 2028

Freedman, W. L., Madore, B. F., Gibson, B. K., et al. 2001, ApJ, 553, 47

Fritz, J., Poggianti, B. M., Bettoni, D., et al. 2007, A\&A, 470, 137

Fritz, J., Moretti, A., Poggianti, B., et al. 2017, ArXiv e-prints, arXiv:1704.05088

Fu, J., Hou, J. L., Yin, J., \& Chang, R. X. 2009, ApJ, 696, 668

Genzel, R., Newman, S., Jones, T., et al. 2011, ApJ, 733, 101

Hibbard, J. E., \& Vacca, W. D. 1997, AJ, 114, 1741

Hirschmann, M., Naab, T., Somerville, R. S., Burkert, A., \& Oser, L. 2012, MNRAS, 419, 3200

Hopkins, P. F., Cox, T. J., Hernquist, L., et al. 2013, MNRAS, 430, 1901

Hung, C.-L., Sanders, D. B., Casey, C. M., et al. 2014, ApJ, 791, 63 Izotov, Y. I., Schaerer, D., Blecha, A., et al. 2006, A\&A, 459, 71 
James, B. L., Tsamis, Y. G., \& Barlow, M. J. 2010, MNRAS, 401, 759

James, B. L., Tsamis, Y. G., Barlow, M. J., Walsh, J. R., \& Westmoquette, M. S. 2013a, MNRAS, 428, 86

James, B. L., Tsamis, Y. G., Barlow, M. J., et al. 2009, MNRAS, 398, 2

James, B. L., Tsamis, Y. G., Walsh, J. R., Barlow, M. J., \& Westmoquette, M. S. 2013b, MNRAS, 430, 2097

Jog, C. J. 1997, ApJ, 488, 642

Kauffmann, G., Heckman, T. M., Tremonti, C., et al. 2003, MNRAS, 346, 1055

Kaviraj, S., Darg, D., Lintott, C., Schawinski, K., \& Silk, J. 2012, MNRAS, 419, 70

Kennicutt, Jr., R. C. 1998a, ARA\&A, 36, 189

-. 1998b, ApJ, 498, 541

Kewley, L. J., \& Ellison, S. L. 2008, ApJ, 681, 1183

Lagos, P., Papaderos, P., Gomes, J. M., Smith Castelli, A. V., \& Vega, L. R. 2014, A\&A, 569, A110

Lagos, P., Telles, E., Muñoz-Tuñón, C., et al. 2009, AJ, 137, 5068

Lagos, P., Telles, E., Nigoche Netro, A., \& Carrasco, E. R. 2012, MNRAS, 427, 740

Larson, R. B., \& Tinsley, B. M. 1978, ApJ, 219, 46

Li, Y., Mac Low, M.-M., \& Klessen, R. S. 2004, ApJL, 614, L29

Liske, J., Lemon, D. J., Driver, S. P., Cross, N. J. G., \& Couch, W. J. 2003, MNRAS, 344, 307

Magrini, L., Vílchez, J. M., Mampaso, A., Corradi, R. L. M., \& Leisy, P. 2007, A\&A, 470, 865

Marcon-Uchida, M. M., Matteucci, F., \& Costa, R. D. D. 2010, A\&A, 520, A35

Metz, M., \& Kroupa, P. 2007, MNRAS, 376, 387

Mihos, J. C., \& Hernquist, L. 1996, ApJ, 464, 641

Moreno, J., Torrey, P., Ellison, S. L., et al. 2015, MNRAS, 448, 1107

Moster, B. P., Macciò, A. V., Somerville, R. S., Naab, T., \& Cox, T. J. 2011, MNRAS, 415, 3750

Okazaki, T., \& Taniguchi, Y. 2000, ApJ, 543, 149

Ostriker, E. C., \& Shetty, R. 2011, ApJ, 731, 41

Overzier, R. A., Heckman, T. M., Schiminovich, D., et al. 2010, ApJ, 710, 979

Poggianti, B. M., Fasano, G., Omizzolo, A., et al. 2016, AJ, 151, 78

Poggianti, B. M., Moretti, A., Gullieuszik, M., et al. 2017, ApJ, 844,48
Prantzos, N., \& Boissier, S. 2000, MNRAS, 313, 338

Proxauf, B., Öttl, S., \& Kimeswenger, S. 2014, A\&A, 561, A10

Pryke, C., Halverson, N. W., Leitch, E. M., et al. 2002, ApJ, 568, 46

Recchi, S., Theis, C., Kroupa, P., \& Hensler, G. 2007, A\&A, 470, L5

Renaud, F., Bournaud, F., \& Duc, P.-A. 2015, MNRAS, 446, 2038

Renaud, F., Bournaud, F., Kraljic, K., \& Duc, P.-A. 2014, MNRAS, 442, L33

Rupke, D. S. N., Kewley, L. J., \& Barnes, J. E. 2010, ApJL, 710, L156

Sánchez, S. F., Kennicutt, R. C., Gil de Paz, A., et al. 2012, A\&A, 538, A8

Schawinski, K., Virani, S., Simmons, B., et al. 2009, ApJL, 692, L19

Schlafly, E. F., \& Finkbeiner, D. P. 2011, ApJ, 737, 103

Schoenmakers, R. H. M., Franx, M., \& de Zeeuw, P. T. 1997, MNRAS, 292, 349

Searle, L., \& Zinn, R. 1978, ApJ, 225, 357

Sengupta, C., Scott, T. C., Dwarakanath, K. S., Saikia, D. J., \& Sohn, B. W. 2014, MNRAS, 444, 558

Spergel, D. N., Bean, R., Doré, O., et al. 2007, ApJS, 170, 377

Tempel, E., Tago, E., \& Liivamägi, L. J. 2012, A\&A, 540, A106

Teyssier, R., Chapon, D., \& Bournaud, F. 2010, ApJL, 720, L149

Toomre, A. 1977, in Evolution of Galaxies and Stellar Populations, ed. B. M. Tinsley \& R. B. G. Larson, D. Campbell, 401

Torrey, P., Cox, T. J., Kewley, L., \& Hernquist, L. 2012, ApJ, 746, 108

Vazdekis, A., Sánchez-Blázquez, P., Falcón-Barroso, J., et al. 2010, MNRAS, 404, 1639

Véron-Cetty, M.-P., \& Véron, P. 2010, A\&A, 518, A10

Weinberg, M. D. 1995, ApJL, 455, L31

Wetzstein, M., Naab, T., \& Burkert, A. 2007, MNRAS, 375, 805

White, S. D. M., \& Rees, M. J. 1978, MNRAS, 183, 341

Wild, V., Rosales-Ortega, F., Falcón-Barroso, J., et al. 2014, A\&A, 567, A132

Zackrisson, E., \& Riehm, T. 2010, Advances in Astronomy, 2010, 735284 